\title{
MIS-MICROSTRUCTURE WITH PERIODIC FIELD ELECTRODES. A NEW TYPE OF DYNAMIC SUPERLATTICE
}

\author{
I.E. Tralle \\ Physical and Technical Institute, Academy of Sciences of the Byelorussian SSR, \\ Zhodinskaja St. 4, 220730-Minsk, USSR
}

(Received May 21, 1990)

\begin{abstract}
The electron movement in thin semiconductor layer adjoining interface semiconductor-insulator in metal-insulator-semiconductor (MIS) microstructure with periodic field electrodes is considered. If a voltage of some sufficiently high frequency is applied to such a structure the effect of this high frequency field on a charge carriers in semiconductor is equivalent, in a sense, to the effect of some time-independent effective potential which is a sequence of deep "dynamic" quantum wells, where the charge carriers are localized. It is shown that the electron resonant tunneling may occur in this structure.
\end{abstract}

PACS numbers: 73.40.-c

As it is known from classical electrodynamics there are no absolute maxima and minima of a potential in the electric field free of charges and, as a consequence, the localization of charged particles in this field is impossible, if one means under localization such a state where the particle with the energy less than some definite value can not leave the bounded region under no initial conditions.

However, as was shown by Gaponov and Miller [1], the localization of the particle in the nonuniform high frequency electromagnetic field is possible. It turns out that under definite conditions the force acting on a particle in nonuniform high frequency field is potential and irrespective of charge sign and a particle could be localized in appearing potential wells. This idea was used by the author in [2], where the electron quantum movement in a semiconductor layer of a metal-insulator-semiconductor (MIS) microstructure with periodic field electrodes was considered. It turns out that if a voltage of some sufficiently high frequency is applied to such a structure the effect of this high frequency field on a charge carriers in semiconductor is in a sense equivalent to some time-independent effective potential which is a sequence of deep "dynamic" quantum wells where the charge carriers are localized. 
Probably, it can be used for construction of a new type of superlattice which could be called dynamic superlattice. The main purpose of the paper is to substantiate the latter proposition and to continue the investigation which has been started in [2].

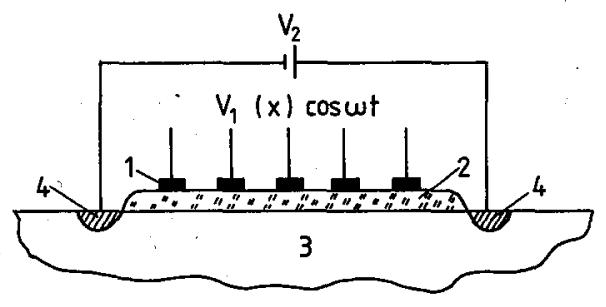

Fig. 1. MIS-microstructure with periodical field electrodes; 1 - field electrodes; 2 insulator; 3 - semiconductor; 4 - the source and the drain.

Suppose we have a MIS-microstructure with the field electrodes which are in the periodic sequence (see Fig. 1) and let the potential applied to the electrodes be of the form: $V(x, t)=V_{1}(x) \times \cos \omega t, V_{1}(x)=V_{1}(x+n \lambda), n$ is the integer. Let the temperature be such that $\lambda<\bar{l}$, where $\bar{l}$ is the electron mean free-path. If one has an indium antimonide as a semiconductor in such a microstructure, the estimations show that at $40 \mathrm{~K}$ the electron mean-free-path in the scattering by phonons is of the order $1.4 \times 10^{-4} \mathrm{~cm}$. On the other hand the modern photolithography makes it possible to get $\lambda$ less than $10^{-4} \mathrm{~cm}$. Also, the choice of semiconductor is determined by the fact that it must have the energy gap as small as possible, so that at temperatures of about several dozens of kelvins there will be sufficient amount of electrons in the conduction band.

Let us consider the electron movement in a thin semiconductor layer adjoining the interface semiconductor-insulator. At the conditions defined above the electron movement along $x$ axis should be described by the one-dimensional Schrödinger equation:

$$
\mathrm{i} \hbar \frac{\partial \Psi}{\partial t}=\left[-\frac{\hbar^{2}}{2 m^{*}} \frac{\partial^{2}}{\partial x^{2}}+e V_{1}(x) \cos \omega t\right] \Psi,
$$

where $e$ and $m^{*}$ are the electron charge and effective mass, respectively. Since $V_{1}(x)$ is a periodic function it could be expanded into Fourier series. The behavior of the electrons in this layer is mainly affected by the lowest harmonics of such series, because the high harmonics are strongly suppressed in the insulator and can be neglected for sufficiently thick insulator [3]. Then $V_{1}(x)$ shall have the form: $V_{1}(x)=\bar{V}_{1}+V_{m} \cos k_{0} x$, where $\bar{V}_{1}$ is a constant component, $k_{0}=2 \pi / \lambda$. Let us take the solution of Eq. (1) in the form [4]:

$$
\Psi(x, t)=\varphi(x, t) \exp \left[-\mathrm{i} e V_{1}(x) \sin \omega t / \hbar \omega\right] .
$$

Substituting (2) into (1) we will have:

$$
\mathrm{i} \hbar \frac{\partial \varphi}{\partial t}=\left[H_{0}+H_{1}(l)+H_{2}(l)\right] \varphi,
$$


where

$$
\begin{gathered}
H_{0}=-\frac{\hbar^{2}}{2 m^{*}} \frac{\partial^{2}}{\partial x^{2}}+\left(4 m^{*} \omega^{2}\right)^{-1}\left(e V_{m} k_{0}\right)^{2} \sin ^{2} k_{0} x \\
H_{1}(t)=-\left(4 m^{*} \omega^{2}\right)^{-1}\left(e V_{m} k_{0}\right)^{2} \sin ^{2} k_{0} x \cos 2 \omega t \\
H_{2}(t)=-\left(\mathrm{i} \hbar / m^{*} \omega\right)\left[\left(e k_{0} V_{m} \sin k_{0} x\right)(\partial / \partial x)+\frac{1}{2}\left(e V_{m} k_{0}^{2} \cos k_{0} x\right)\right] \sin \omega t .
\end{gathered}
$$

Let us make some estimations. Suppose $\lambda \approx 10^{-4} \mathrm{~cm}, k_{0}=2 \pi \times 10^{4} \mathrm{~cm}^{-1}$, $m^{*} \approx 0.02 m_{e}, \omega \approx 35 \times 10^{8} \mathrm{~Hz}, V_{m}=0.01 \mathrm{~V}$.

Then we have:

$$
\max \left|H_{1}(l)\right| \sim\left(4 m^{*} \omega^{2}\right)^{-1}\left(e V_{m} k_{0}\right)^{2} \approx 10^{-15} \mathrm{~J} .
$$

Supposing $\partial \varphi / \partial x \sim 1 / \Delta x=1 / \lambda$, we get

$$
\max \left|H_{2}(\grave{l})\right| \sim \frac{\hbar e k_{0} V_{m}}{m^{*} \omega \lambda}+\frac{\hbar e V_{m} k_{0}^{2}}{2 m^{*} \omega} \approx 9.6 \times 10^{-20} \mathrm{~J} .
$$

Hence, in subsequent analysis we restrict our consideration to the first terms on the right-hand side of Eq. (3), neglecting $\mathrm{H}_{2}(t)$.

We carry out the analysis of the equation obtained in this way in two steps: first, we consider the equation with time-independent right-hand side (i.e. with the Hamiltonian $H_{0}$ ), and then find out the consequence of the existence of the $H_{1}(t)$ term. We have the equation then:

$$
\mathrm{i} \hbar \frac{\partial \varphi}{\partial t}=\left[-\frac{\hbar^{2}}{2 m^{*}} \frac{\partial^{2}}{\partial x^{2}}+\left(4 m^{*} \omega^{2}\right)^{-1}\left(e V_{m} k_{0}\right)^{2} \sin ^{2} k_{0} x\right] \varphi .
$$

Introducing new variables $y=k_{0} x$ and $\varphi(y, t)=\bar{\varphi}(y) \exp \left(-\mathrm{i} \hbar^{-1} \varepsilon_{n} t\right)$.we obtain:

$$
\frac{d^{2} \bar{\varphi}}{d y^{2}}+\left(a_{n}+2 q \cos 2 y\right) \bar{\varphi}=0 .
$$

Here $a_{n}=-\beta\left(1-\varepsilon_{n} / \alpha\right), q=\beta / 2=m^{*} \alpha / \hbar^{2} k_{0}^{2}$ are dimensionless quantities; $\alpha=$ $\left(e V_{m} k_{0}\right)^{2} / 8 m^{*} \omega^{2}$ and $\varepsilon_{n}$ are the quantities which have the sense and dimension of energy.

Equation (4) is the Mathieu equation, the parameter $\dot{a}_{n}$, considered as a function of $q$, is the eigenvalue related to the corresponding Mathieu function.

At the above values of $\lambda, k_{0}, m^{*}$ we obtained for $q$ the value $q \approx 9.2 \times 10^{8}$, and hence at so large $q$ one could use the asymptote of the eigenvalues [5, 6]:

$$
a_{n} \sim-2|q|+2(2 n+1)|q|^{1 / 2}, \quad n=0,1,2, \ldots
$$

and for the energy eigenvalues we have:

$$
\varepsilon_{n}=(2 n+1) q^{-1 / 2} .
$$

Note that the obtained spectrum is equidistant with the distance between the levels $\Delta \varepsilon_{n, n-1}=2 q^{-1 / 2} \alpha$, which for the chosen values of parameters is equal to $\Delta \varepsilon=3.66 \times 10^{-20} \mathrm{~J}=0.228 \mathrm{eV}$. 
Let us consider now the solution of the equation with time-dependent $H_{1}(t)$ term. In accordance with common quantum-mechanical rules [7], the solutions should be of the form:

$$
\varphi=\sum_{n} b_{n}(t) \bar{\varphi}_{n}^{(0)} .
$$

Here $\bar{\varphi}_{n}^{(0)}$ are the Mathieu functions, and the time-dependent coefficients $b_{n}(t)$ are obeying the system of equations:

$$
\mathrm{i} \hbar \dot{b}_{n}(l)=\sum_{m} H_{n m}^{(1)}(t) b_{m}(l)
$$

where

$$
H_{n m}^{(1)}(t)=\int_{0}^{\pi(2 \pi)} \bar{\varphi}_{n}^{(0)} H_{1}(t) \bar{\varphi}_{m}^{(0)} \mathrm{d} y
$$

Depending on that Mathieu functions are $\pi$-or $2 \pi$-periodic, in the last integral the upper limit is $\pi$ or $2 \pi$.

Using the orthogonality and normalization of Mathieu functions [5], one can prove that $H_{n m}^{(1)}=0$, if $\bar{\varphi}_{n}^{(0)}$ and $\bar{\varphi}_{m}^{(0)}$ are of different parity. parity.

Let us consider more carefully the case when $\bar{\varphi}_{n}^{(0)}$ and $\bar{\varphi}_{m}^{(0)}$ are of the same

Let

$$
\begin{aligned}
& \bar{\varphi}_{n}^{(0)}=c e_{2 n}(y,-q)=(-1)^{n} \sum_{r=0}^{\infty}(-1)^{r} A_{2 r}^{(2 n)} \cos 2 r y, \\
& \bar{\varphi}_{m}^{(0)}=c e_{2 m}(y,-q)=(-1)^{m} \sum_{r=0}^{\infty}(-1)^{r} A_{2 r}^{2 m} \cos 2 r y .
\end{aligned}
$$

Using the orthogonality of the functions again one can prove that $H_{n m}^{(1)}=\alpha f_{n m} \cos 2 \omega l$, where

$$
\begin{gathered}
f_{n m}=\frac{1}{2} \sum_{r=0}^{\infty} \sum_{s=0}^{\infty}(-1)^{r+s} A_{2 r}^{(2 n)} A_{2 s}^{(2 m)}\left(\delta_{r-1, s}+\delta_{r+1, s}\right)= \\
=-\frac{1}{2}\left(A_{2}^{(2 n)} A_{0}^{(2 m)}+A_{4}^{(2 n)} A_{2}^{(2 m)}+\ldots+A_{0}^{(2 n)} A_{2}^{(2 m)}+A_{2}^{(2 n)} A_{4}^{(2 m)}+\ldots\right) .
\end{gathered}
$$

The evaluation of the sum in the brackets requires a special care, but one can note that asymptotic behavior of $A_{2 r}^{(2 n)}$ at $q \rightarrow \infty$ does not depend on the upper index. For example (see [5]): $\lim _{q \rightarrow \infty}\left(A_{2}^{(2 n)} / A_{0}^{(2 n)}\right)=-2, \lim _{q \rightarrow \infty}\left(A_{2 r}^{(2 n)} / A_{0}^{(2 n)}\right)=$ $(-1)^{r} 2$ at arbitrary $n$.

Using the reccurence formula for the $c e_{2 n}$ :

$$
a A_{0}^{(2 n)}-q A_{2}^{(2 n)}=0,
$$

one can demonstrate that for $q=1600$ the ratio $A_{2} / A_{0}=-1.95$ for the $c e_{0}$ function and -1.75 for $c e_{2}$ function. For $q=9.2 \times 10^{8}$ we have $A_{2} / A_{0}=-1.999934$ for $c e_{0}$ and -1.99934 for $c e_{2}$ functions, respectively. 
Hence, we can conclude that $f_{n m} \approx-\sum_{s} A_{2 s}^{(2 n)} A_{2(s-1)}^{(2 m)}$. Owing to the fact that the series of Mathieu functions (7) is absolutely convergent, one can conclude that the latter sum does not exceed some finite quantity $\delta$. Hence we have a next system of equations for $b_{n}(t)$ coefficients:

$$
\mathrm{i} \hbar \dot{b}_{n}(t)=\alpha \delta \cos 2 \omega t \sum_{i} b_{i}(t)
$$

Let us note that the $A_{2 s}^{(2 n)}$ coefficients possess another asymptote, namely $A_{2 s}^{(2 n)} \rightarrow 0$ for $n \rightarrow \infty$ and $n>q$. We can restrict ourselves to a finite number of terms on the right-hand side of (8), e.g. $N$. Summing up we have:

$$
\mathrm{i} \hbar \hat{\dot{b}}=(\alpha \delta / N) \cos (2 \omega t) \widehat{b},
$$

where $\hat{b}=\left(b_{1}, b_{2}, \ldots, b_{N}\right)$. The solution of $(9)$ is:

$$
\widehat{b}=\widehat{c}(0) \exp [(-\mathrm{i} / 2 \hbar N) \alpha \delta \sin 2 \omega t],
$$

where $\widehat{c}(0)=\left(c_{1}(0), c_{2}(0), \ldots, c_{N}(0)\right)$ is the matrix consisting of coefficients at the initial time $t=0$.

It follows that $\int\left|\varphi \varphi^{*}\right| \mathrm{d} y=\sum_{n}\left|c_{n}(0)\right|^{2}=1$, i.e. despite of the fact that the Hamiltonian in Eq. (3) is explicitely time-dependent and the energy, generally speaking, should not conserve, but the asymptotic properties of Mathieu functions caused by a large value of $q$ make the electrons be in the states with the energies $\varepsilon_{n}$ and probabilities, corresponding to their initial distribution.

The physical explanation consists of the fact that, since $\omega \ll \Delta \varepsilon_{n, n-1} / \hbar$, the perturbation $H_{1}(t)$ must be regarded as adiabatic, but as it is known from quantum.mechanics [8] the adiabatic perturbation can not cause the transitions between the states of discrete spectrum. So, the existence of the $H_{1}(t)$ term in Hamiltonian results only in appearing the new phase factor of the wave function and hence we can regard the electron as moving in some effective time-independent potential $U_{\text {eff }}=2 \alpha \sin ^{2} k_{0} x$.

We will show that the negative differential conductivity could take place in such a microstructure. First of all, let us make some qualitative comments. We will consider the consequences of barriers and the incident particles with the energies smaller than the height of the barriers. These barriers are practically impenetrable for almost all particle energies, however for certain discret energies and respective energy level width the particles can pass through the barriers without any reflexion. This is the resonant tunneling phenomenon $[9,10]$.

The stationary equation (4) can be rewritten in the form:

$$
\begin{gathered}
\frac{\hbar^{2}}{2 m^{*}} \Psi^{\prime \prime}+(\varepsilon-u(x)) \Psi=0 \\
u(x)= \begin{cases}2 \alpha \sin ^{2} k_{0} x-e|E| x & \text { for } x \in[0, L] \\
0 & \text { for } \quad x<0, x>L .\end{cases}
\end{gathered}
$$


Here $\boldsymbol{E}$ is the intensity connected with $V_{2}$ by the relation $e|\boldsymbol{E}| L=V_{2}, V_{2}$ is the voltage applied between the source and the drain, and $L$ is the total length of the structure.

The tunneling problem consists of finding all the solutions of Eq. (10) parametrically dependent on $\varepsilon \in(0, \infty)$ and behaving as:

$$
\Psi(k, x)= \begin{cases}\exp (\mathrm{i} k x)+r(k) \exp (-\mathrm{i} k x), & \text { for } \quad x<0, \\ t(k) \exp (\mathrm{i} k x) & \text { for } x>L ;\end{cases}
$$

(the case of normalized particle beam incident from the left); $k^{2}=\varepsilon ; r(k), t(k)$ stand for reflexion and transmission amplitudes.

We will now introduce the outgoing propagator $G^{+}\left(x, x^{\prime} ; k\right)$ and define the electron wave function along the internal region $0 \leq x \leq L$ by $\Psi(k, x)=$ $2 \mathrm{i} k G^{+}(0, x ; k)$. Then the transmission amplitude is $t(k)=2 \mathrm{i} k G^{+}(0, L ; k) \exp (-\mathrm{i} k L)$. Near an isolated pole $k_{n}=\alpha_{n}-\mathrm{i} \gamma_{n}$ it is possible to write the propagator in the form $[11,12]$ :

$$
G^{+}\left(x, x^{\prime} ; k\right) \approx \frac{\varphi_{n}(x) \varphi_{n}\left(x^{\prime}\right)}{2 k\left(k-k_{n}\right)} .
$$

Here $\varphi_{n}(x)$ are the eigenfunctions of the Iramiltonian on the left-hand side of Eq. (10), $k_{n}^{2}=\varepsilon_{n}^{\prime}-\mathrm{i} \Gamma_{n} / 2, \varepsilon_{n}^{\prime}=\alpha_{n}^{2}-\gamma_{n}^{2}, \Gamma_{n}=4 \alpha_{n} \gamma_{n}$. Using (11) and laking into account that $\alpha_{n} \gg \gamma_{n}$, for the transmission coefficient $|\ell(k)|^{2}$ we have:

$$
|\ell(k)|^{2}=\frac{k^{2}\left|\varphi_{n}(0)\right|^{2}\left|\varphi_{n}(L)\right|^{2}}{\alpha_{n}^{2}\left[\left(k-\alpha_{n}\right)^{2}+\gamma_{n}^{2}\right]}
$$

Let us notice that in accordance with [12] $\gamma_{n}=\left(\left|\varphi_{n}(0)\right|^{2}+\left|\varphi_{n}(L)\right|^{2}\right) / 2 I$, and $I \approx 1$.

Let the potential in (10) possesses only two maxima (it consists of two barriers), i.e. $L=2 \pi / k_{0}$. As far as $2 \alpha \gg e|E| L$, with high accuracy we can consider the barriers to be symmetric, that leads to $\left|\varphi_{n}(0)\right|^{2}=\left|\varphi_{n}(L)\right|^{2}$ and the latter involve $\lim _{k \rightarrow \alpha_{n}\left(\varepsilon \rightarrow \varepsilon_{n}^{\prime}\right)}|l(k)|^{2}=1$.

Now let the number of identical barriers be $m>2$. As it was proved in [13], if any arbitrary pair of barriers is completely transparent at some encrgy $\varepsilon=\varepsilon_{n}^{\prime}$, then all barriers composed of $m>2$ replicas are also completely transparent at this energy.

Thus the barrier transmission coefficient has the maxima at the electron energies $\varepsilon \rightarrow \varepsilon_{n}^{\prime}$. So far as the current amplitude $I \sim|t(k)|^{2}$, then the curent vs. voltage ( $I-V$ characteristics) can have a number of sharp peaks divided by valleys and this means an availability of negative differential conductivity.

So we can conclude that the character of current flow in this MIS-microstructure at the conditions formulated above in some features is similar to carrier transport in ordinary (static) superlattice with resonant tunreling.

\section{References}

[1] A.V. Gaponov, M.A. Miller, Sov. Phys. JETP 34, 242 (1958). 
[2] I.E. Tralle, Dokl. $A N$ BSSR 32, 1081 (1988) (in Russian).

[3] U. Wulf, Phys. Rev. B 35, 9754 (1987).

[4] R.J. Cook, D.G. Shancland, A.L. Wells, Phys. Rev. A 31, 564 (1985).

[5] N.W. McLachlan, Theory and Application of Mathieu Functions, Oxford 1947.

[6] H. Bateman, A. Erdéyi, Higher Transcendental Functions, Vol. 3, Mc Graw-IIll, New York 1955.

[7] L.D. Landau, E.M. Lifshitz, Quantum Mechanics, Nauka, Moscow 1974.

[8] A.S. Davydov, Quantum Mechanics, Nauka, Moscow 1973.

[9] E. Burstein, S. Lundquist, eds., Tunneling Phenomena in Solids, Plenum Press, New York 1969.

[10] L. Tsu, L. Esaki, Appl. Phys. Lelt. 22, 562 (1973).

[11] G. Garcia-Calderon, R.E. Peierls, Nucl. Phys. A 265, 443 (1976).

[12] G. Garcia-Calderon, Solid State Commun. 62, 441 (1987).

[13] J. Kowalski, J.L. Fry, J. Malh. Phys. 22, 2407 (1987). 